\title{
ЭФФЕКТИВНОСТЬ ВНУТРИКОСТНОГО ВВЕДЕНИЯ АУТОЛОГИЧНОЙ ОБОГАЩЕННОЙ ТРОМБОЦИТАМИ ПЛАЗМЫ В ЗОНУ ОТЕКА КОСТНОГО МОЗГА ПРИ ОСТЕОАРТРОЗЕ КОЛЕННОГО СУСТАВА
}

\author{
А. В. Лычагин, А. В. Гаркави, О. И. Ислейих , П. И. Катунян, Д. С. Бобров, Р. Х. Явлиева, Е. Ю. Целищева
}

Первый Московский государственный медицинский университет имени И. М. Сеченова (Сеченовский Университет), Москва, Россия

\begin{abstract}
Остеоартроз (ОА) поражает как пожилых людей, для которых он одна из основных причин инвалидности, так и лиц трудоспособного возраста и является актуальной клинической и социальной проблемой ввиду устойчивости болевого синдрома к проводимой терапии. Заболеванию характерна деструкция внутрисуставных и параартикулярных структур, таких как субхондральная кость. При ОА важным признаком патологических изменений служит отек костного мозга (ОКМ). В работе рассмотрены вопросы влияния ОКМ на развитие гонартроза, а также терапевтические подходы к ведению пациентов с ОА. Целью исследования была разработка методики лечения ОКМ при ОА коленного сустава путем локального внутрикостного введения в зону отека аутологичной обогащенной тромбоцитами плазмы (PRP). Исследовали 17 пациентов с диагнозом “Остеоартроз II-IV ст.» по классификации Kellgren-Lawrence, у которых на МРТ в субхондральной зоне выявлены области локального воспаления в виде ОКМ в соответствии с международной классификацией WORMS. Средний возраст пациентов составил 41,7 $\pm 14,3$ лет. Пациентам внутрикостно из внесуставного доступа в зону ОКМ вводили аутологичную обогащенную тромбоцитами плазму под рентгеноскопическим контролем. Оценку эффективности лечения проводили по шкалам ВАШ, WOMAC и KOOS до введения аутоплазмы, через 1 и 3 месяца после начала лечения. Через 3 месяца после манипуляции отмечалось статистически значимое снижение показателей интенсивности воспалительного синдрома: по WOMAC на 17,5\%, KOOS на 19,4\% и по BAШ на 33,1\% (p < 0,01). Таким образом, доказана эффективность внутрикостного введения аутологичной обогащенной тромбоцитами плазмы в лечении пациентов с ОА, сопровождающимся ОКМ в субхондральной зоне.
\end{abstract}

Ключевые слова: отек костного мозга, остеоартроз, аутологичная обогащенная тромбоцитами плазма, внутрикостное введение, качество жизни

Информация о вкладе авторов: А. В. Лычагин и О. И. Ислейих - планирование исследования, подбор литературы, интерпретация данных, подготовка черновика рукописи; А. В. Гаркави — планирование исследования, интерпретация данных; П. И. Катунян — планирование исследования, подготовка черновика рукописи; Д. С. Бобров, Р. Х. Явлиева и Е. Ю. Целищева — планирование исследования.

Соблюдение этических стандартов: исследование одобрено локальным комитетом по этике ФГАОУ ВО Первый МГМУ имени И. М. Сеченова (протокол № 06-18 от 06 июня 2018 г.). Все пациенты подписали добровольное информированное согласие.

Для корреспонденции: Осама Ибрахим Ислейих

ул. Большой Тишинский переулок, д. 26/15, г. Москва, 123557; osaibso@yahoo.com

Статья получена: 12.07.2019 Статья принята к печати: 26.07.2019 Опубликована онлайн: 20.08.2019

DOI: $10.24075 /$ vrgmu.2019.053

\section{EFFECTIVENESS OF INTRAOSSEOUS INFILTRATION OF AUTOLOGOUS PLATELET-RICH PLASMA IN THE AREA OF THE BONE MARROW EDEMA IN OSTEOARTHRITIS OF THE KNEE JOINT}

Lychagin AV, Garkavi AV, Islaieh OI $₫$, Katunyan PI, Bobrov DS, Yavlieva RH, Tselisheva EYu

I.M. Sechenov First Moscow State Medical University, Moscow, Russia

Osteoarthritis (OA) affects both elderly people, for whom it is one of the main causes of disability, and people of active working age and is an urgent clinical and social problem of resistance of pain syndrome to therapy. The disease is characterized by both destruction of intra-articular and paraarticular structures, such as subchondral bone. While OA is an important sign of pathological changes believe the bone marrow edema (BME). This work examines the effect of BME on development osteoarthritis, and therapeutic approaches to the management of patients with OA. The aim of the study was to develop a method of treatment of BME in OA of the knee joint by locally intraosseous injection of autologous thrombotic-rich plasma (PRP) into the edema zone. In this study 17 patients with the diagnosis: Osteoarthritis II-IV Grade. according to the classification of Kellgren-Lawrence, in which areas of local inflammation in the form of BME were detected on MRI in the subchondral zone in accordance with the international classification of WORMS (Whole Organ Magnetic Resonance Imaging Score). The mean age of patients was $41,7 \pm 14,3$ years, 10 of them were women and 7 men. Patients were treated with autological platelet-rich plasma under $x$-ray control injected from extra-articular intraosseous access in the area of BME. Evaluation of effectiveness of treatment performed by VAS, WOMAC and KOOS scales, before the introduction of autoplasma, after 1 and 3 months after the start of treatment. Three months after the manipulation, there was a statistically significant decrease in the intensity of inflammatory syndrome: for WOMAC by $17.5 \%$, for KOOS by $19.4 \%$ and for VAS by $33,1 \%(p<0,01)$. Thus, the efficiency of intraosseous Infiltration of autologous platelet-rich plasma in the treatment of patients with $\mathrm{OA}$, accompanied by edema of the bone marrow in the subchondral zone, was proved.

Keywords: bone marrow edema, osteoarthritis, autologous platelet-rich plasma, intraosseous Infiltration, quality of life

Author contribution: Lychagin AV and Islaieh $\mathrm{OI}$ — research planning, literature collection and analysis, data interpretation, draft preparation; Garkavi AV — research planning, data interpretation; Katunyan PI — research planning, draft preparation; Bobrov DS, Yavlieva RH and Tselisheva EYu — research planning.

Compliance with ethical standards: the study was approved by the local ethics committee of the Sechenov University (protocol № 06-18 dated June 06, 2018). All patients agreed to participate in the study in writing.

$\square$ Correspondence should be addressed: Osama I. Islaieh

Bolshoi Tishinsky Pereulok 26/15, Moscow, 123557; osaibso@yahoo.com

Received: 12.07.2019 Accepted: 26.07.2019 Published online: 20.08.2019

DOI: 10.24075/brsmu.2019.053

По данным ВОЗ, частота встречаемости остеоартроза (ОА) в настоящее время составляет 11-13\% населения мира. ОА поражает как пожилых людей, для которых он является одной из основных причин инвалидности, так и лиц трудоспособного возраста [1-4]. Заболевание характеризуется хронической болью, деструкцией и потерей суставного хряща, ремоделированием субхондральной кости, образованием остеофитов, воспалением синовиальной оболочки различной степени, вовлечением в патологический процесс как внутрисуставных, так и параартикулярных структур [5].

Долгое время доминирующую роль в развитии ОА отводили суставному хрящу, однако в последнее десятилетие 
растет интерес к роли субхондральной кости (СК): с точки зрения как этиопатогенеза, так и клинического значения [6] Доказано, что ремоделирование СК представляет собой важный процесс в патогенезе ОА [7]. Изменения в ней могут развиваться первично, в качестве пускового механизма ОА или вторично, в результате нарастания дегенеративнодистрофических процессов [8-10].

Важным признаком патологических изменений СK при ОА служит отек костного мозга (ОКМ), определяемый на манитно-резонансной томограмме (МРТ). Этот термин был впервые применен в 1988 г., и его все чаще используют для описания патологического МР-сигнала, определяемого при ОА [11, 12]. ОКМ обнаруживают обычно в зоне склероза СК, его сопровождают увеличение доли объема костной ткани и уплотнение трабекулярного слоя [13]. При прогрессировании ОА зона ОКМ нередко увеличивается, что считают важным фактором риска для дальнейшего прогрессирования деструкции суставных структур [2, 14, 15]. Показано, что ОКМ и деформация сустава могут быть предикторами быстрого прогрессирования ОА [4]. Более того, очаговые повреждения хряща часто находятся в непосредственной близости к ОКМ, и степень деструкции хрящевой ткани коррелирует с ростом интенсивности МР-сигнала [16, 17]. Ряд исследователей считают ОКМ одной из причин развития выраженного болевого синдрома. У пациентов с ОА, предъявлявших жалобы на сильные боли в области пораженного сустава, ОКМ с площадью на МРТ более $1 \mathrm{~cm}^{2}$ встречался значительно чаще, чем в тех случаях, когда симптомы были не столь выражены [14, 18]. У больных, имеющих ОКМ, отмечали значимое прогрессирование деструкции хряща и появление болевого синдрома [19]. Таким образом, ОКМ можно считать предиктором начала деградации хряща и появления боли еще до манифестации всех типичных клинических признаков ОА. В то же время ряд авторов считает, что ОКМ может ассоциироваться не только с ОА, но и с остеонекрозом, сопровождающимся болями в покое [20, 21].

Несмотря на то что влиянию ОКМ на течение ОА уделяют в последнее время много внимания, единого мнения относительно роли ОКМ еще не сформировано. Особенно много вопросов остается в отношении возможности воздействия на патологический субхондральный очаг в процессе комплексного лечения ОА. Важность изменений СК стала более очевидной, когда пришло понимание связи между нею и суставным хрящом. Эта связь получила название остеохондральной функциональной единицы. Было показано, что ОКМ тесно связан с прогрессированием дегенерации внутрисуставных структур и усилением боли в суставе. Таким образом, увеличение ОКМ повышает вероятность эндопротезирования в качестве оптимальной лечебной тактики $[22,23]$.

Генетический и гистологический анализ трепанобиоптатов зоны показал, что боль линейно коррелирует не только с прогрессированием ОА, но и с изменениями в микросреде субхондрального ОКМ [24]. В зонах ОКМ отмечена высокая метаболическая активность с экспрессией генов, участвующих в воспалительных процессах [24]. Предполагают, что ОКМ представляет собой локальную область высокого костного метаболизма с повышенным накоплением цитокинов и ангиогенных факторов, приводящих к росту новых сосудов и нервных окончаний в этой области [25].

Известен метод субхондропластики, применяемый для лечения костно-хрящевой патологии суставов при остеоартрозе, когда в пораженную субхондральную кость вводят фоссрат кальция под артроскопическим контролем. Эффективность этой процедуры была показана на примере лечения 133 пациентов с гонартрозом в сочетании с OKM, хотя через 2,5 года после выполненной субхондропластики $25 \%$ пациентов не отметили улучшения и согласились на эндопротезирование сустава [23]. Хороший эффект субхондропластики показан и в другом исследовании, где 70\% из 164 пациентов с гонартрозом с показаниями к эндопротезированию после проведенного лечения отметили такое улучшение, что отказались от эндопротезирования [26].

Все большее распространение получает группа методик, основанных на внутрикостном введении обогащенной тромбоцитами аутоплазмы [27-29]. Продолжается изучение механизмов, обусловливающих хорошие клинические результаты при инъекционном введении аутологичной обогащенной тромбоцитами плазмы (PRP), однако уже сейчас общепризнанны противовоспалительный и регенераторный эффеекты такой терапии. В связи с этим попытка создания методики лечения ОА, основанной на введении в очаг отека костного мозга PRP, представляется перспективной.

Целью исследования была разработка терапевтического подхода к лечению ОКМ при ОА коленного сустава путем локального внутрикостного введения в зону отека аутологичной обогащенной тромбоцитами плазмы.

\section{ПАЦИЕНТЫ И МЕТОДЫ}

В исследовании участвовали 17 пациентов $(41,7 \pm 14,3$ лет), 15 из них с ОА коленного сустава II-IV ст. по классификации Kellgren-Lawrence, у которых, по данным MPТ, была обнаружена зона локального воспаления в виде ОКМ с преимущественным поражением медиальных отделов коленного сустава [30]. Критерии включения: пациенты обоих полов в возрасте от 40 до 80 лет; преобладание артроза коленного сустава; боль в суставах выше 3 баллов по ВАШ; рентгенологические степени тяжести 2 и 4 по классификации I. Kellgren и I. Lawrence с отеком костного мозга в субхондральной зоне; индекс массы тела - 20-33; возможность для наблюдений во время всего периода исследования; психическая адекватность, способность и готовность к сотрудничеству и выполнению рекомендаций врача. Критерии исключения: двусторонний артроз коленных суставов с синовитом; индекс массы тела > 33; полиартрит; тяжелая деформация конечности (варусное искривление диафиза более $4{ }^{\circ} \mathrm{C}$ и вальгусное более $\left.16{ }^{\circ} \mathrm{C}\right) ;$ артроскопия менее года до начала лечения; внутрисуставное введение гиалуроновой кислоты в течение последних 6 месяцев; системные аутоиммунные заболевания; плохо контролируемый сахарный диабет (гликозилированный гемоглобин выше 9\%); заболевания крови (коагулопатии, анемия с НВ < 90); проведение иммуносупрессивной терапии, введение варфарина или других антикоагулянтов; лечение кортикостероидами в течение 6 месяцев до включения в исследование; отказ пациента от дальнейшего участия в исследовании; выявление объективных противопоказаний к операции; отсутствие возможности динамического наблюдения и контроля в течение установленного срока.

Длительность заболевания составила от 1 до 9 лет $(5,2 \pm 4,5)$. Диагноз ОА устанавливали на основании жалоб, данных анамнеза и клинико-рентгенологического обследования. Всем пациентам выполнена рентгенография коленного сустава в двух проекциях: передне-задней и боковой, при сгибании голени под углом $30^{\circ} \mathrm{C}$ (табл. 1). 
У всех пациентов оценивали состояние субхондральной зоны и OKM по данным МРТ. Для описания отека костного мозга использовали международную классификацию WORMS (Whole Organ Magnetic Resonance Imaging Score), основанную на увеличении интенсивности сигнала Т2 в последовательности изображений [2]. Оценку проводили по балльной шкале, измеряя максимальный диаметр очага отека на срезе MPT с помощью программы RadiAnt DICOM Viewer 4.6.9 (64-bit) (Medixant; Франция). Критерии диагноза представлены в табл. 2. На рис. 1 и 2 представлень примеры томограмм пациентов с тяжелым ОКМ.

У всех пациентов при поступлении оценивали выраженность болевого синдрома по ВАШ, а также определяли сумму баллов по функциональным шкаламопросникам WOMAC и KOOS [31-33]. Эти же параметры определяли и далее, в ходе динамического наблюдения через 1 и 3 месяца.

\section{Подготовка препарата PRP и описание метода}

По методике Regenlab по технологии REGEN ACR (Regen Lab SA; Швейцария) получали препарат, который определенное время сохраняется после внутрикостного введения: 30 мл аутологичной венозной крови распределяли по трем пробиркам - две пробирки REGEN BCT (для получения аутологичной богатой тромбоцитами плазмы) и одну пробирку REGEN ATS (для получения аутологичной тромбиновой сыворотки, которую используют для активации препарата) (шаг 1). Все пробирки центрифугировали в течение 5 мин со скоростью 3100 об./мин. (шаг 2). Затем в стерильных условиях из REGEN BCT в шприц набирали PRP, и из REGEN ATS - аутологичную тромбиновую сыворотку в соотношении $10: 1$ (шаг 3) (рис. 2). Внутрикостное введение обогащенной тромбоцитами плазмы осуществляли в зону OKM, определенную ранее с помощью МРТ в режиме Т2: в медиальный или латеральный мыщелки бедренной или большеберцовой кости. Манипуляцию проводили в стандартном положении пациента лежа на спине на операционном столе, под внутривенной анестезией (шаг 4) (рис. 2).
Для введения препарата использовали стилет с четырехгранным мандреном 13 (Stryker; США), под контролем электронно-оптического преобразователя (ЭОП). После достижения иглой патологического очага в него плавно вводили 5 мл изготовленного препарата (рис. 3).

В ближайшем периоде после введения препарата всем пациентам рекомендовали: местное применение холодных компрессов; ограничение нагрузки до 1 недели; ограничение повышенной нагрузки до 2 недель; при возникновении болей - перорально парацетамол до 4 г/сут. Селективные нестероидные противовоспалительные препараты не использовали.

Статистическую обработку полученных данных выполняли при помощи STATISTICA 13.3 (StatSoft; CШA).

\section{РЕЗУЛЬТАТЫ ИССЛЕДОВАНИЯ}

По данным МРТ, минимально выраженный ОКМ выявлен у 2 пациентов, умеренно выраженный - у 7, тяжелый - у 8 (рис. 4).

После введения PRP в динамике отмечали значимое снижение болевого синдрома по шкале ВАШ. Перед началом лечения пациенты определяли боль как «сильную» (51,4 \pm 6,9 баллов), через месяц боль снизилась на 36,4 балла, перейдя в диапазон «незначительной» $(15,0 \pm 8,3, p<0,01)$, а через 3 месяца показатель ВАШ составил 18,3 \pm 11,6 баллов $(p<0,01)$, что также является показателем «незначительной» боли (рис. 5).

По шкале WOMAC также отмечено существенное улучшение показателей. Средняя сумма баллов при поступлении составила 57,38 $\pm 12,85$, через месяц после введения препарата она была равна 76,45 \pm 5,91 балла ( $p<0,01)$, а через 3 месяца достигла 75,33 \pm 8,41 $(p<0,01)$ (рис. 5).

Аналогичная динамика результатов была отмечена и по шкале KOOS: среднее значение при поступлении составило 52,78 \pm 13,38 балла, а через месяц после введения препарата $-72,00 \pm 7,35$ балла $(p<0,01)$, через 3 месяца достигло 72,13 \pm 8,50 балла ( $<<0,01)$ (рис. 5).

Поскольку шкала KOOS состоит из 5 разделов, оценивающих различные аспекты состояния коленного

Таблица 1. Характеристика пациентов

\begin{tabular}{|c|c|c|}
\hline \multirow{2}{*}{ Пол (количество пациентов) } & Женский & $10(58,8 \%)$ \\
\hline & Мужской & $7(41,2 \%)$ \\
\hline \multicolumn{2}{|l|}{ Средний возраст (лет) } & $41,7 \pm 14,3$ \\
\hline \multicolumn{2}{|l|}{ Длительность поражения коленного сустава (лет) } & $5,2 \pm 4,5$ \\
\hline \multicolumn{2}{|l|}{ Средний срок наблюдения (месяцев) } & $5,5 \pm 2,5$ \\
\hline Пораженный сустав (количество) & Односторонний & $17(100 \%)$ \\
\hline \multirow{4}{*}{ Рентгенологическая стадия по шкале Kellgren-Lawrence } & 1 & 0 \\
\hline & II & $5(29,4 \%)$ \\
\hline & III & $10(58,8 \%)$ \\
\hline & IV & $2(11,8 \%)$ \\
\hline
\end{tabular}

Таблица 2. Критерии диагноза по шкале WORMS

\begin{tabular}{|l|c|c|}
\hline \multicolumn{1}{|c|}{ Степень OKM } & Диаметр очага (мм) & Баллы по шкале WORMS \\
\hline Отсутствие & <5 & 0 \\
\hline Минимально выраженный & $5-20$ & 1 \\
\hline Умеренно выраженный & $>20$ & 2 \\
\hline Тяжелый & 3 \\
\hline
\end{tabular}


сустава, представляет интерес их оценка по отдельности (табл. 3). По всем параметрам была отмечена достоверная положительная динамика относительно начала терапии. Следует отметить, что наиболее выраженное улучшение среднего показателя зафиксировано по разделам «спорт и отдых» (от 25,83 \pm 21 до 53,33 \pm 28,86) и «качество жизни» (от $24,08 \pm 18,39$ до 54,18 $\pm 21,48)$ к третьему месяцу. Болевой синдром уменьшался к первому месяцу, затем незначительно нарастал к третьему месяцу, но его значения все еще свидетельствовали о достоверном улучшении состояния.

Важно отметить, что по большинству разделов субшкалы KOOS, а также по шкалам WOMAC и BAШ наилучшие показатели отмечены через месяц после введения препарата, а к 3 месяцам средние показатели немного ухудшались, хотя это различие не всегда было статистически значимым.

\section{ОБСУЖДЕНИЕ РЕЗУЛЬТАТОВ}

Разработанная нами методика внутрикостного введения препарата PRP в субхондральную зону является малоинвазивным доступным способом лечения гонартроза

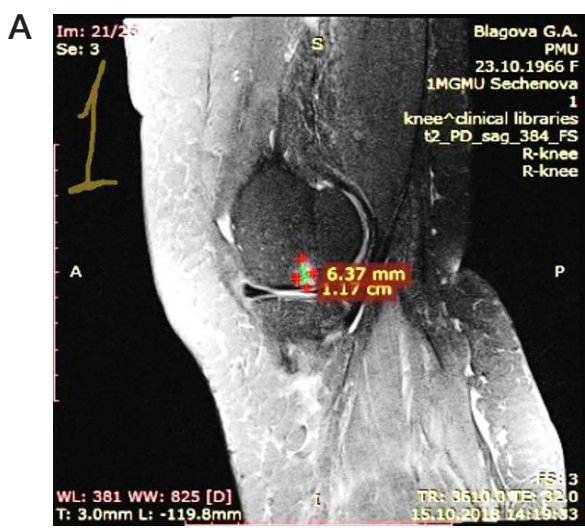

с отеком костного мозга. Применяемый препарат PRP, изготовленный по технологии REGENACR, обладает пролонгированным лечебным действием. Ранее было показано, что при ОКМ образуется локальная область высокого костного метаболизма с повышенным накоплением цитокинов и ангиогенных факторов, что является по сути локальным воспалением [24, 25]. Положительная динамика состояния пациентов на фоне применения PRP поддерживает теорию о выраженном противовоспалительном эффекте местного применения обогащенной тромбоцитами плазмы. Несмотря на то что PRP содержит ангиогенные и просибротические факторы роста, не было зарегистрировано ни одного случая ухудшения клинического течения заболевания.

Наши данные во многом согласуются с данными других авторов, применявших PRP внутрикостно в лечении гонартроза [27-29]. У 14 пациентов с тяжелым гонартрозом при трехкратном применении внутрисуставных инъекций (8 мл PRP) в сочетании с субхондральными внутрикостными инъекциями (5 мл PRP) в медиальный мыщелок большеберцовой кости и медиальный мыщелок бедра через 6 месяцев, как и в нашей работе, получено

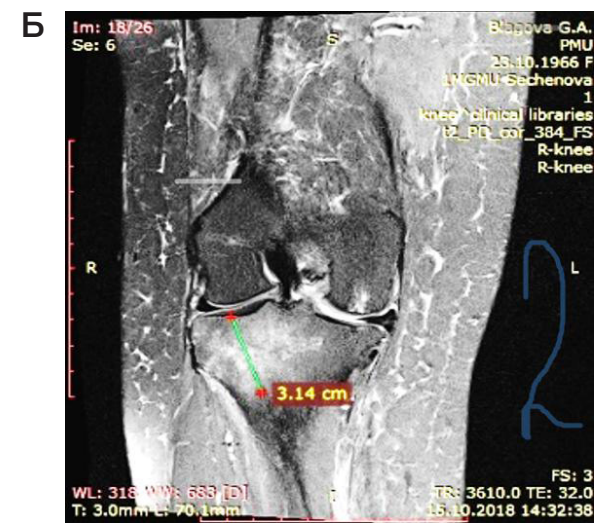

Рис. 1. Оценка размеров OKM по классификации WORMS с помощью программы RadiAnt DICOM Viewer 4.6.9 (64-bit). A. OKM в латеральном мыщелке бедренной кости размером 1,17 см. Б. ОКМ в медиальном мыщелке большеберцовой кости размером 3,14 см

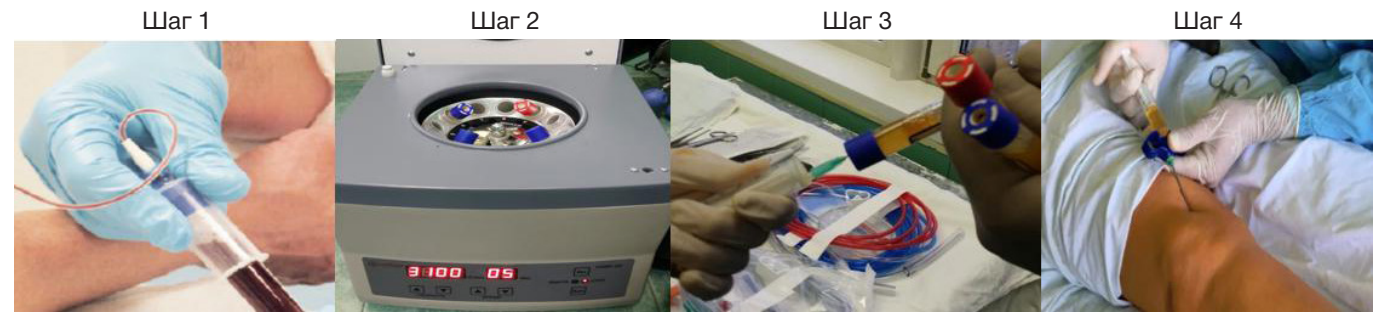

Рис. 2. Подготовка препарата PRP. Шаг 1: забор крови. Шаг 2: центрифугирование. Шаг 3: смешивание препарата в пропорции 10 : 1. Шаг 4: использование готового препарата

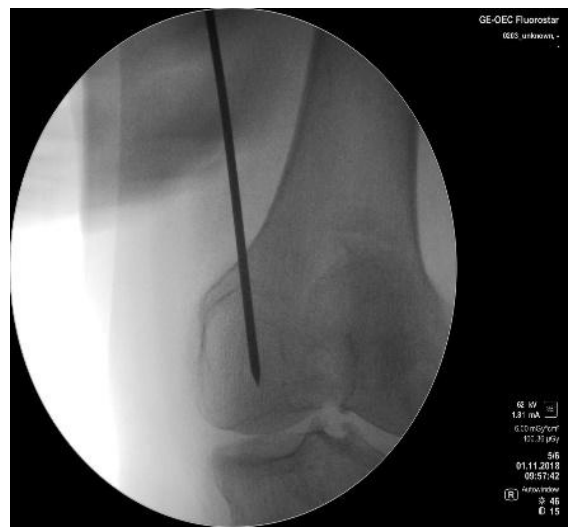

Рис. 3. Введение препарата PRP в зону OKM в медиальный мыщелок бедренной кости под контролем ЭОП. На снимке инъекционная игла находится во внутреннем мыщелке бедренной кости 
статистически значимое снижение боли и других показателей по шкале KOOS [29]. В нашем случае препарат вводили внутрикостно один раз, при этом клиническое улучшение было отмечено на 3 месяца раньше.

Тем не менее необходимо признать, что к 3 месяцам после внутрикостного введения препарата достигнутый эффект снижается, хотя сохраняется достоверное улучшение результатов клинических тестов.

Наиболее выраженное улучшение среднего показателя по шкале KOOS в разделах «спорт и отдых» и «качество жизни», по-видимому, обусловлено эмоциональной составляющей пациентов, почувствовавших положительный эффект от проведенного лечения, и требует допольнительных способов оценки.

\section{ВЫВОДЫ}

При остеоартрозе II-IV ст. по классификации KellgrenLawrence в субхондральной зоне на МРТ-исследовании выявляют очаги ОКМ, являющиеся одним из звеньев патогенеза заболевания, способствующие его дальнейшему прогрессированию и поддерживающие болевой синдром. Введение аутологичной обогащенной тромбоцитами плазмы в очаг ОКМ оказывает выраженный и стойкий

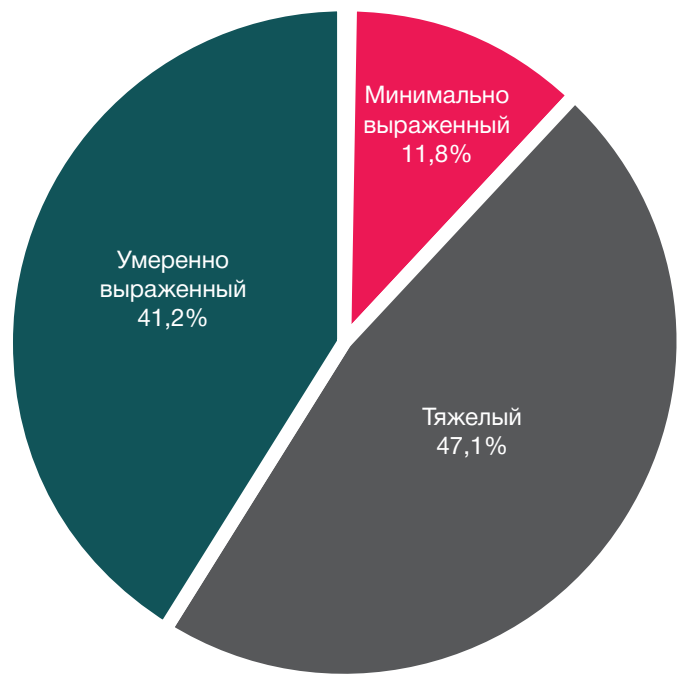

Рис. 4. Распределение пациентов по степени выраженности OKM по классификации WORMS

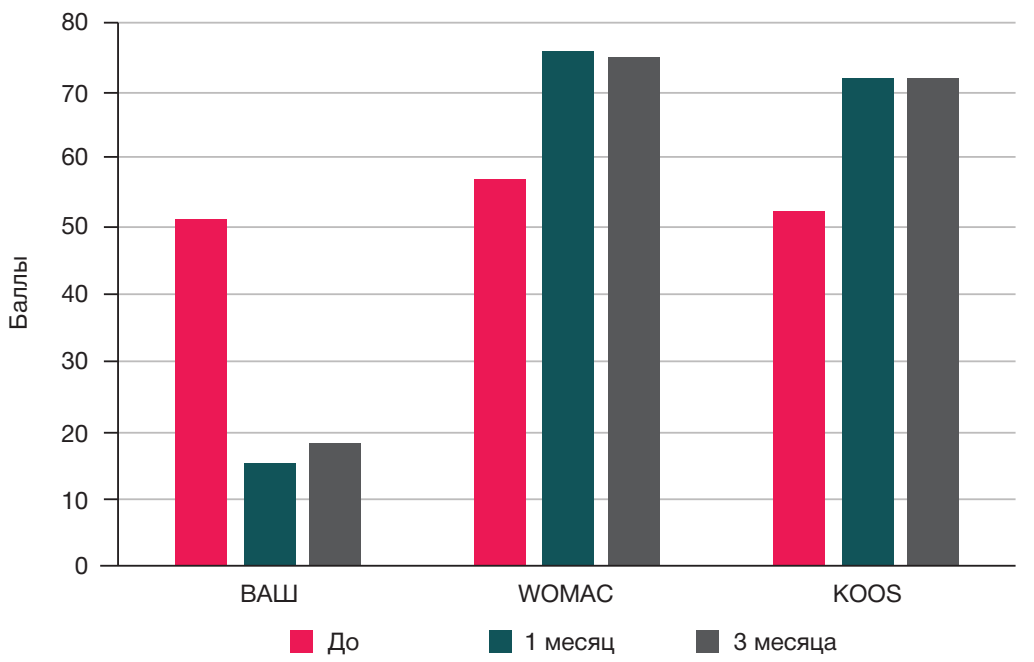

Рис. 5. Динамика средних показателей по шкалам ВАШ, WOMAC и KOOS. В исследовании 17 пациентам с остеоартрозом коленного сустава была введена PRP в зоне OKM. Состояние оценивали до начала терапии, а также через 1 и 3 месяца после проведенного лечения

Таблица 3. Оценка результатов функционального состояния по субшкалам KOOS

\begin{tabular}{|l|c|c|c|}
\hline & До & 1 месяц после операции месяца после операции \\
\hline \multicolumn{1}{|c|}{ Симптомы } & $62,85 \pm 10,28$ & $74,28 \pm 10,53^{*}$ & $71,43 \pm 6,18^{*}$ \\
\hline Боль & $53,70 \pm 7,18$ & $74,40 \pm 11,87^{*}$ & $70,36 \pm 12,52^{*}$ \\
\hline Активность & $53,36 \pm 15,41$ & $58,33 \pm 19,66^{*}$ & $74,51 \pm 4,24^{*}$ \\
\hline Спорт и отдых & $25,83 \pm 21$ & $40,62 \pm 23,30^{*}$ & $53,33 \pm 28,86{ }^{*}$ \\
\hline Качество жизни & $24,08 \pm 18,39$ & $72,00 \pm 7,35^{*}$ & $72,13 \pm 8,50^{*}$ \\
\hline Итоговый индекс & $52,78 \pm 13,38$ & $21,48^{*}$ \\
\hline
\end{tabular}

Примечание: * - статистически значимое изменение параметра по сравнению с исходными данными $(p<0,01)$. 
положительный эффект в виде существенного снижения боли и улучшения функции пораженного сустава, который сохраняется как минимум до 3 месяцев.

Дальнейшее изучение метода лечения пациентов с ОКМ при гонартрозе, основанного на использовании обогащенной тромбоцитами аутоплазмы, является важной перспективной задачей современной ортопедии ввиду положительной динамики состояния пациентов и полученных данных о важности остеохондральной функциональной единицы в патологическом процессе.

\section{Литература}

1. Felson DT, McLaughlin S, Goggins J, LaValley MP, Gale ME, Totterman S, et al. Bone marrow edema and its relation to progression of knee osteoarthritis. Ann Intern Med. 2003; (139): 330-6.

2. Kazakia GJ, Kuo D, Schooler J, Siddiqui S, Shanbhag S, Bernstein G et al. Arthritis Research \& Therapy. 2013; (15): 11-2.

3. Meizer R, Radda C, Stolz G, Kotsaris S, Petje G, Krasny C, et al. $\mathrm{MRI}$-controlled analysis of 104 patients with painful bone marrow edema in different joint localizations treated with the prostacyclin analogue iloprost. Wien Klin Wochenschr. 2005; (117): 278-86.

4. Зайцева Е. М. Оценка минеральной плотности костной ткани субхондральных отделов бедренной и большеберцовой костей при гонартрозе. Научно-практическая ревматология. 2005; (1): 27-30.

5. Felson DT. An update on the pathogenesis and epidemiology of osteoarthritis. Radiol Clin North Am. 2004; (42): 1-9.

6. Алексеева Л. И., Зайцева Е. М. Роль субхондральной кости при остеоартрозе. НИИ ревматологии РАМН. Научнопрактическая ревматология. М., 2009; (4): 43-8.

7. Delgado D, Garate A, Vincent H, Bilbao AM, Patel R, Fiz N, at al. Current concepts in intraosseous Platelet-Rich Plasma injections for knee osteoarthritis. Journal of Clinical Orthopaedics and Trauma. 2019; (10): 36-4.

8. Roemer FW, Frobell R, Hunter DJ, Crema MD, Fischer W, Bohndorf $\mathrm{K}$, et al. MRI-detected subchondral bone marrow signal alterations of the knee joint: terminology, imaging appearance, relevance and radiological differential diagnosis. Osteoarthritis Cartilage. 2009; (17): 1115-31.

9. Roemer FW, Neogix T, Nevittk MC, Felsonx DT, Zhux Y, Zhangx Y, et al. Subchondral bone marrow lesions are highly associated with and predict subchondral bone attrition longitudinally. The MOST study Osteoarthritis and Cartilage. 2010; (18): 47-53.

10. Sowers MF, Hayes C, Jamadar D, Capul D, Lachance L, Jannausch M, et al. Magnetic resonance-detected subchondral bone marrow and cartilage defect characteristics associated with pain and X-ray-defined knee osteoarthritis. Osteoarthritis and Cartilage. 2003; (6): 387-393.

11. Wilson AJ, Murphy WA, Hardy DC, Totty WG. Transient osteoporosis: transient bone marrow edema? Radiology. 1988; (167): 757-760.

12. Lecouvet FE, van de Berg BC, Maldague BE, Lebon CJ, Jamart J, Saleh M, et al. Early irreversible osteonecrosis versus transient lesions of the femoral condyles: prognostic value of subchondral bone and marrow changes on MR imaging. AJR Am J Roentgenol. 1998; (170): 71-7.

13. Hunter DJ, Gerstenfeld L, Bishop G, Davis AD, Mason ZD, Einhorn TA, et al. Bone marrow lesions from osteoarthritis knees are characterized by sclerotic bone that is less well mineralized. Arthritis Res Ther. 2009; (11): 11.

14. Manicourt DH, Brasseur JP, Boutsen Y, Depreseux G, Devogelaer JP. Role of alendronate in therapy for posttraumatic complex regional pain syndrome type I of the lower extremity. Arthritis Rheum. 2004; (50): 3690-97.

15. Pelletier JP, Raynauld JP, Berthiaume MJ, Abram F, Choquette D, Haraoui B, et al. Risk factors associated with the loss of cartilage volume on weight-bearing areas in knee osteoarthritis patients assessed by quantitative magnetic resonance imaging: a longitudinal study. Arthritis Research and Therapy. 2007; (4): 74.

16. Zhao J, Li X, Bolbos RI, Link TM, Majumdar S. Longitudinal assessment of bone marrow edema-like lesions and cartilage degeneration in osteoarthritis using 3 T MR T1rho quantification. Skeletal Radiol. 2010: (39): 523-31.

17. Carrino JA, Blum J, Parellada JA, Schweitzer ME, Morrison WB.
MRI of bone marrow edema-like signal in the pathogenesis of subchondral cysts. Osteoarthritis Cartilage. 2006; (14): 1081-15.

18. Peterfy CG, Guermazi A, Zaim S, Tirman PF, Miaux Y, White D, et al. Whole-Organ Magnetic Resonance Imaging Score (WORMS) of the knee in osteoarthritis. Osteoarthritis and Cartilage. 2004; 12 (3): 177-90.

19. Wluka AE, Hanna F, Davies-Tuck M, Wang Y, Bell RJ, Davis SR, et al. Bone marrow lesions predict increase in knee cartilage defects and loss of cartilage volume in middle-aged women without knee pain over 2 years. Ann Rheum Dis. 2009; (68): 850-5.

20. lida $S$, et al. Correlation between bone marrow edema and collapse of the femoral head in steroid-induced osteonecrosis. AJR. American Journal of Roentgenology. 2000; 174 (3): 735-43.

21. Ito $H$, Matsuno $T$, Minami $A$. Relationship between bone marrow edema and development of symptoms in patients with osteonecrosis of the femoral head. AJR. American Journal of Roentgenology. 2006; 186 (6): 1761-70.

22. Perry T, O'Neill T, Parkes M, Felson DT, Hodgson R, Arden NK. Bone marrow lesion type and pain in knee osteoarthritis. Ann Rheum Dis. 2018; (77): 1145.

23. Tanamas SK, Wluka AE, Pelletier JP, Pelletier JM, Abram F, Berry $\mathrm{PA}$, et al. Bone marrow lesions in people with knee osteoarthritis predict progression of disease and joint replacement. A longitudinal study Rheumatology. 2010; (49): 2413-19.

24. Berger CE, Kroner AH, Minai-Pour MB, Ogris E, Engel A. Biochemical markers of bone metabolism in bone marrow edema syndrome of the hip. Bone. 2003; (33): 346-51.

25. Kuttapitiya A, Assi L, Laing K, Hing C, Mitchell P, Whitley G, et al. Microarray analysis of bone marrow lesions in osteoarthritis demonstrates upregulation of genes implicated in osteochondral turnover, neurogenesis and inflammation. Ann Rheum Dis. 2017; 76 (10): 1764-73

26. Astur DC, de Freitas EV, Cabral PB, Morais CC, Pavei BS, Kaleka CC, et al. Evaluation and management of subchondral calcium phosphate injection technique to treat bone marrow lesion Cartilage. 2018; (10): 1177

27. Su K, Bai Y, Wang J, Zhang H, Liu H, Ma S. Comparison of hyaluronic acid and PRP intra-articular injection with combined intra-articular and intraosseous PRP injections to treat patients with knee osteoarthritis. 2018; (37): 1341-50.

28. Fiz N, Pérez JC, Guadilla J, Garate A, Sánchez P, Padilla S, et al. Intraosseous Infiltration of Platelet-Rich Plasma for Severe Hip Osteoarthritis. 2017; 19 (6): 821-5.

29. Sánchez M, Anitua E, Delgado D, Sanchez P, Prado R, Goiriena JJ, et al. A new strategy to tackle severe knee osteoarthritis: Combination of intra-articular and intraosseous injections of Platelet Rich Plasma. Expert Opinion on Biological Therapy. 2016; (10): 15-7.

30. Kellgren JH, JeVrey M, Ball J. Atlas of standard radiographs. Vol 2. Oxford: Blackwell Scientific, 1963.

31. Roos E, Lohmander L. The Knee injury and Osteoarthritis Outcome Score (KOOS): from joint injury to osteoarthritis. Health and Quality of Life Outcomes. 2003; 1 (1): 64-72.

32. Marot V, Murgier J, Carrozzo A, Reina N, Monaco E, Chiron P et al. Determination of normal KOOS and WOMAC values in a healthy population. Knee Surgery, Sports Traumatology, Arthroscopy. 2018; 27 (2): 541-8.

33. Hawker G, Mian S, Kendzerska T, French M. Measures of adult pain: Visual Analog Scale for Pain (VAS Pain), Numeric Rating Scale for Pain (NRS Pain), McGill Pain Questionnaire (MPQ), Short-Form McGill Pain Questionnaire (SF-MPQ), Chronic Pain Grade Scale (CPGS), Short Form-36 Bodily Pain Scale (SF. Arthritis Care \& Research. 2011; 63 (S11): S240-52. 
1. Felson DT, McLaughlin S, Goggins J, LaValley MP, Gale ME, Totterman S, et al. Bone marrow edema and its relation to progression of knee osteoarthritis. Ann Intern Med. 2003; (139): 330-6.

2. Kazakia GJ, Kuo D, Schooler J, Siddiqui S, Shanbhag S, Bernstein G et al. Arthritis Research \& Therapy. 2013; (15): 11-2.

3. Meizer R, Radda C, Stolz G, Kotsaris S, Petje G, Krasny C, et al. $\mathrm{MRI}$-controlled analysis of 104 patients with painful bone marrow edema in different joint localizations treated with the prostacyclin analogue iloprost. Wien Klin Wochenschr. 2005; (117): 278-86.

4. Zajceva EM, Smirnov AV, Alekseeva I. Ocenka mineral'noj plotnosti kostnoj tkani subhondral'nyh otdelov bedrennoj i bol'shebercovoj kostej pri gonartroze. Nauchno-prakticheskaja revmatologija. 2005; (1): 27-30.

5. Felson DT. An update on the pathogenesis and epidemiology of osteoarthritis. Radiol Clin North Am. 2004; (42): 1-9.

6. Alekseeva LI, Zajceva EM. Rol' subhondral'noj kosti pri osteoartroze. NII revmatologii RAMN, Moskva. Nauchno-prakticheskaja revmatologija. 2009; (4): 43-8.

7. Delgado D, Garate A, Vincent H, Bilbao AM, Patel R, Fiz N, at al. Current concepts in intraosseous Platelet-Rich Plasma injections for knee osteoarthritis. Journal of Clinical Orthopaedics and Trauma. 2019; (10): 36-4.

8. Roemer FW, Frobell R, Hunter DJ, Crema MD, Fischer W, Bohndorf $\mathrm{K}$, et al. MRl-detected subchondral bone marrow signal alterations of the knee joint: terminology, imaging appearance, relevance and radiological differential diagnosis. Osteoarthritis Cartilage. 2009; (17): 1115-31.

9. Roemer FW, Neogix T, Nevittk MC, Felsonx DT, Zhux Y, Zhangx Y, et al. Subchondral bone marrow lesions are highly associated with and predict subchondral bone attrition longitudinally. the MOST study Osteoarthritis and Cartilage. 2010; (18): 47-53.

10. Sowers MF, Hayes C, Jamadar D, Capul D, Lachance L, Jannausch M, et al. Magnetic resonance-detected subchondral bone marrow and cartilage defect characteristics associated with pain and X-raydefined knee osteoarthritis. Osteoarthritis and Cartilage. 2003; (6): 387-393.

11. Wilson AJ, Murphy WA, Hardy DC, Totty WG. Transient osteoporosis: transient bone marrow edema? Radiology. 1988; (167): 757-760.

12. Lecouvet FE, van de Berg BC, Maldague BE, Lebon CJ, Jamart J, Saleh $\mathrm{M}$, et al. Early irreversible osteonecrosis versus transient lesions of the femoral condyles: prognostic value of subchondral bone and marrow changes on MR imaging. AJR Am J Roentgenol. 1998; (170): 71-7.

13. Hunter DJ, Gerstenfeld L, Bishop G, Davis AD, Mason ZD, Einhorn TA, et al. Bone marrow lesions from osteoarthritis knees are characterized by sclerotic bone that is less well mineralized. Arthritis Res Ther. 2009; (11): 11.

14. Manicourt DH, Brasseur JP, Boutsen Y, Depreseux G, Devogelaer JP. Role of alendronate in therapy for posttraumatic complex regional pain syndrome type I of the lower extremity. Arthritis Rheum. 2004; (50): 3690-97.

15. Pelletier JP, Raynauld JP, Berthiaume MJ, Abram F, Choquette D, Haraoui B, et al. Risk factors associated with the loss of cartilage volume on weight-bearing areas in knee osteoarthritis patients assessed by quantitative magnetic resonance imaging: a longitudinal study. Arthritis Research and Therapy. 2007; (4): 74.

16. Zhao J, Li X, Bolbos RI, Link TM, Majumdar S. Longitudinal assessment of bone marrow edema-like lesions and cartilage degeneration in osteoarthritis using 3 T MR T1rho quantification. Skeletal Radiol. 2010: (39): 523-31.

17. Carrino JA, Blum J, Parellada JA, Schweitzer ME, Morrison WB.
MRI of bone marrow edema-like signal in the pathogenesis of subchondral cysts. Osteoarthritis Cartilage. 2006; (14): 1081-15.

18. Peterfy CG, Guermazi A, Zaim S, Tirman PF, Miaux Y, White D, et al. Whole-Organ Magnetic Resonance Imaging Score (WORMS) of the knee in osteoarthritis. Osteoarthritis and Cartilage. 2004; 12 (3): 177-90.

19. Wluka AE, Hanna F, Davies-Tuck M, Wang Y, Bell RJ, Davis SR, et al. Bone marrow lesions predict increase in knee cartilage defects and loss of cartilage volume in middle-aged women without knee pain over 2 years. Ann Rheum Dis. 2009; (68): 850-5.

20. lida $\mathrm{S}$, et al. Correlation between bone marrow edema and collapse of the femoral head in steroid-induced osteonecrosis. AJR. American Journal of Roentgenology. 2000; 174 (3): 735-43.

21. Ito $H$, Matsuno $T$, Minami $A$. Relationship between bone marrow edema and development of symptoms in patients with osteonecrosis of the femoral head. AJR. American Journal of Roentgenology. 2006; 186 (6): 1761-70.

22. Perry T, O'Neill T, Parkes M, Felson DT, Hodgson R, Arden NK. Bone marrow lesion type and pain in knee osteoarthritis. Ann Rheum Dis. 2018; (77): 1145.

23. Tanamas SK, Wluka AE, Pelletier JP, Pelletier JM, Abram F, Berry PA, et al. Bone marrow lesions in people with knee osteoarthritis predict progression of disease and joint replacement. A longitudinal study Rheumatology. 2010; (49): 2413-19.

24. Berger CE, Kroner AH, Minai-Pour MB, Ogris E, Engel A. Biochemical markers of bone metabolism in bone marrow edema syndrome of the hip. Bone. 2003; (33): 346-51.

25. Kuttapitiya A, Assi L, Laing K, Hing C, Mitchell P, Whitley G, et al. Microarray analysis of bone marrow lesions in osteoarthritis demonstrates upregulation of genes implicated in osteochondral turnover, neurogenesis and inflammation. Ann Rheum Dis. 2017; 76 (10): 1764-73.

26. Astur DC, de Freitas EV, Cabral PB, Morais CC, Pavei BS, Kaleka CC, et al. Evaluation and management of subchondral calcium phosphate injection technique to treat bone marrow lesion Cartilage. 2018; (10): 1177.

27. Su K, Bai Y, Wang J, Zhang H, Liu H, Ma S. Comparison of hyaluronic acid and PRP intra-articular injection with combined intra-articular and intraosseous PRP injections to treat patients with knee osteoarthritis. 2018; (37): 1341-50.

28. Fiz N, Pérez JC, Guadilla J, Garate A, Sánchez P, Padilla S, et al. Intraosseous Infiltration of Platelet-Rich Plasma for Severe Hip Osteoarthritis. 2017; 19 (6): 821-5.

29. Sánchez M, Anitua E, Delgado D, Sanchez P, Prado R, Goiriena JJ, et al. A new strategy to tackle severe knee osteoarthritis: Combination of intra-articular and intraosseous injections of Platelet Rich Plasma. Expert Opinion on Biological Therapy. 2016; (10): 15-7.

30. Kellgren $\mathrm{JH}$, JeVrey M, Ball J. Atlas of standard radiographs. Vol 2. Oxford: Blackwell Scientific, 1963.

31. Roos E, Lohmander L. The Knee injury and Osteoarthritis Outcome Score (KOOS): from joint injury to osteoarthritis. Health and Quality of Life Outcomes. 2003; 1 (1): 64-72.

32. Marot V, Murgier J, Carrozzo A, Reina N, Monaco E, Chiron P et al. Determination of normal KOOS and WOMAC values in a healthy population. Knee Surgery, Sports Traumatology, Arthroscopy. 2018; 27 (2): 541-8.

33. Hawker G, Mian S, Kendzerska T, French M. Measures of adult pain: Visual Analog Scale for Pain (VAS Pain), Numeric Rating Scale for Pain (NRS Pain), McGill Pain Questionnaire (MPQ), Short-Form McGill Pain Questionnaire (SF-MPQ), Chronic Pain Grade Scale (CPGS), Short Form-36 Bodily Pain Scale (SF. Arthritis Care \& Research. 2011; 63 (S11): S240-52. 